\title{
Extending the Ring Theory of Personhood to the Care of Dying Patients in Intensive Care Units
}

\section{Natalie Pei Xin Chan, et al. [full author details at the end of the article]}

Received: 15 June 2021 / Revised: 17 September 2021 / Accepted: 23 September 2021 /

Published online: 20 October 2021

(c) The Author(s) 2021

\begin{abstract}
It is evident, in the face of the COVID-19 pandemic that has physicians confronting death and dying at unprecedented levels along with growing data suggesting that physicians who care for dying patients face complex emotional, psychological and behavioural effects, that there is a need for their better understanding and the implementation of supportive measures. Taking into account data positing that effects of caring for dying patients may impact a physician's concept of personhood, or "what makes you, 'you'", we adopt Radha Krishna's Ring Theory of Personhood (RToP) to scrutinise the experiences of physicians working in intensive care units (ICU) using a fictional scenario that was inspired by real events. The impact of death and dying, its catalysts, internal constituents, external factors, dyssynchrony, and buffers, specific to ICU physicians, were identified and explored. Such a framework allows for ramifications to be considered holistically and facilitates the curation of strategies for conflict resolution. This evaluation of the RToP acknowledges the experience and wide-ranging effects it has on ICU physicians. As such, our findings provide insight into their specific needs and highlight the importance of support on a personal and organisational level. Although further research needs to be conducted, the RToP could serve as the basis for a longitudinal assessment tool supported by the use of portfolios or mentorship due to their provision of personalised, appropriate, specific, timely, accessible and long-term support.
\end{abstract}

Keywords COVID-19 $\cdot$ Intensive care unit (ICU) $\cdot$ Death and dying $\cdot$ Personhood · Ring theory of personhood (RToP)

$\begin{array}{ll}\text { Abbreviations } \\ \text { COVID-19 } & \text { Coronavirus disease } 2019 \\ \text { ICU } & \text { Intensive Care Unit } \\ \text { RToP } & \text { Ring Theory of Personhood } \\ \text { ACP } & \text { Advance Care Plan }\end{array}$




\section{Background}

The COVID-19 pandemic has seen medical professionals taking to social media sites to share their knowledge and experiences with dying patients (Ouyang 2020a). Not only were these physicians educating their colleagues and the public on clinical developments surrounding the COVID-19 infections, they also provided rare insights into the human aspects of caring for the dying during this trying time (Engelhart and Smith 2020; Ouyang 2020b; Schmidt 2020). Perhaps nowhere has the impact of death and dying on physicians been as evident as in the intensive care unit (ICU) where stress, manpower shortages, evolving care conditions and complex treatment decisions (Barnett et al. 2016; Brooks et al. 2017b; Donnelly and Psirides 2015; Laurent et al. 2017; Pattison et al. 2013; Trankle 2014; Wåhlin et al. 2010)—such as limiting care for the aged, those with poor mobility, cancer and other life-limiting illnesses-have created emotional, psychological, moral and existential distress amongst physicians (Engelhart and Smith 2020; Ouyang 2020b; Schmidt 2020).

Indeed, so profound have these effects been that fundamental changes in the thoughts (Aslakson et al. 2010; Monteiro et al. 2016; Simmonds 1996; Svantesson et al. 2003), decision-making (Aslakson et al. 2010; Monteiro et al. 2016; Simmonds 1996; Svantesson et al. 2003) and relationships (Almansour et al. 2019; Amati and Hannawa 2014; Asch et al. 1995; Baggs et al. 2012; Brooks et al. 2017b; Chikhladze et al. 2016; Cohen et al. 2005; Gutierrez 2012; Hamric and Blackhall 2007; Hawryluck et al. 2002; Henrich et al. 2016; Hough et al. 2005; Laurent et al. 2017; Monteiro et al. 2016; Simmonds 1996; Trankle 2014; Wåhlin et al. 2010; Weng et al. 2011; Workman et al. 2003) of ICU physicians have been reported. In addition, how these physicians have come to view their professional responsibilities, and how they perceive the meeting of their professional duties and interactions, have evolved (Aslakson et al. 2010; Brooks et al. 2017a, 2017b; Donnelly and Psirides 2015; Festic et al. 2012; Hamric and Blackhall 2007; Jensen et al. 2013; Laurent et al. 2017; McAndrew and Leske 2015; Mehter et al. 2018; Nordgren and Olsson 2004; Robertsen et al. 2019; Svantesson et al. 2003; Trankle 2014; Wåhlin et al. 2010; Zambrano et al. 2012).

In this study, we use Radha Krishna's definition of personhood, or "what makes you, you" (Radha Krishna et al. 2015; Radha Krishna and Alsuwaigh 2015, 1), from the Ring Theory of Personhood (RToP). With such wide ramifications reported, it might be said that the personhood of ICU physicians have also been altered (Daly et al. 2018; Henrich et al. 2016; Nordgren and Olsson 2004; Robertsen et al. 2019). The significance of such a hypothesis is vast. The notion that such experiences can shake and change the very foundations of a physician's personhood (Begley 2020; Oliver 2020; Phua et al. 2020) suggests that addressing its effects will likely require a comprehensive approach. As a result, better understanding of these effects upon their personhood is required (Henrich et al. 2016). 


\section{Current Understanding of Personhood}

To discuss this issue holistically, we adopt Radha Krishna's RToP (Radha Krishna et al. 2015; Radha Krishna and Alsuwaigh 2015), a clinically evidenced holistic concept of personhood created within the palliative care setting. Aside from being situated in the context of care for the dying, the RToP has also been shown to capture evolving concepts of personhood in changing conditions over time which would mirror anticipated changes in the ICU setting.

Radha Krishna's RToP conceives of personhood as four interconnected, concentric rings. These are referred to as the innate, individual, relational and societal rings (Fig. 1).

The Innate Ring comprises inalienable aspects of all humans. This status is conferred to each person by virtue of their connections with the divine or their genetic makeup which endows them with human features, for example their blood type which cannot be altered. The combination of all these considerations confers the individual respect, considerations and rights reserved for all human beings till death. The Innate Ring also considers aspects that a person is born into or with. These include their gender, ethnicity, facial features, skin and hair colour, family set up, familial, religious and cultural beliefs as well as their very culture and society. These features may be altered and are thus considered to be changeable aspects or the Secondary Innate Ring. By virtue of its association with religious and cultural belief systems, the Secondary Innate Ring contains beliefs, values and principles that the

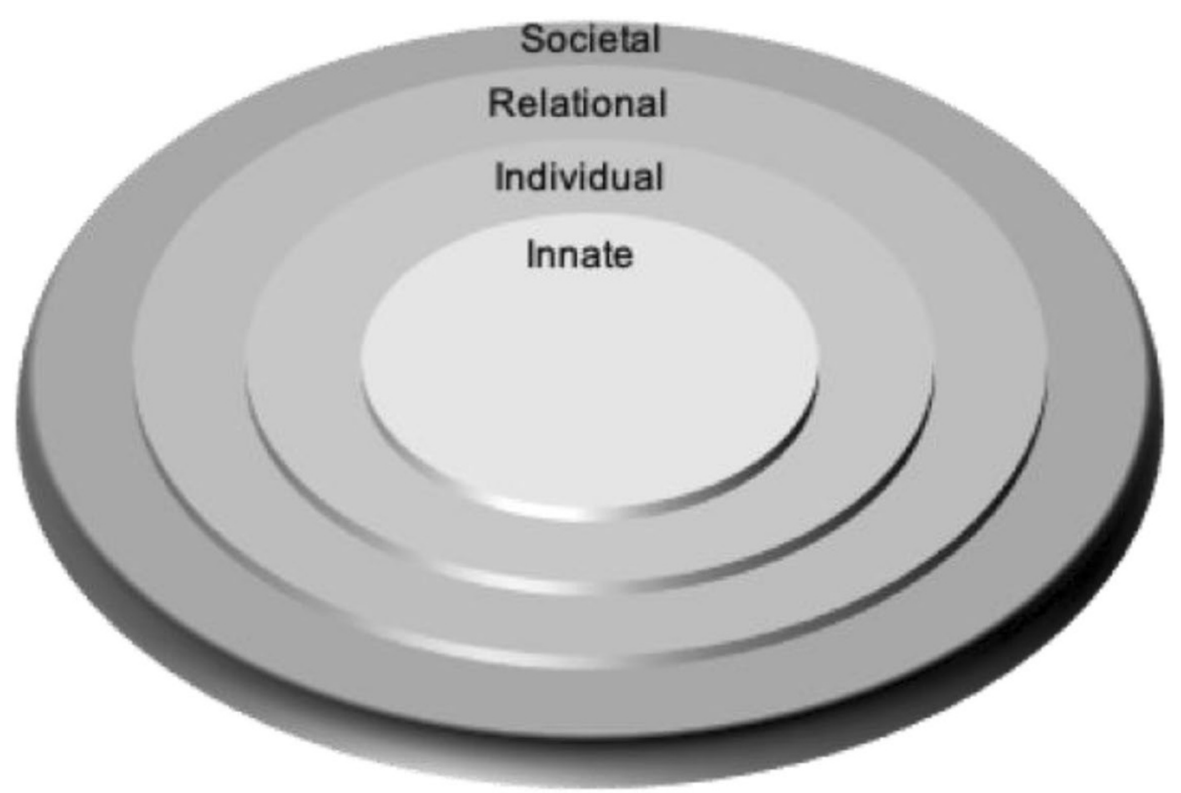

Fig. 1 Ring Theory of Personhood. Radha Krishna's Ring Theory of Personhood - personhood is conceived as four interconnected, concentric rings: innate, individual, relational and societal rings 
person abides by. As with all the rings, these influence thinking, preferences, decision-making, interactions and behaviours.

The Individual Ring is built upon the Innate Ring and relates to a person's conscious functions. These include their ability for cogitation, communication, self-awareness and action. By virtue of their thoughts, preferences and experiences, there are also beliefs, values, motivations and principles rooted in the Individual Ring. These may overlap with those of the Innate Ring which are rooted in familial, cultural and religious beliefs highlighting the interconnectedness of the rings. The RToP's ability to detect change in the Individual Ring will help to better understand the impact on ICU physicians' thinking, emotions and coping (Dyregrov and Gjestad 2011; Youngblut et al. 2017).

The Relational Ring comprises of close personal relationships as defined by the individual, and mutually reciprocated by the other party. There are some relationships that are apparent from birth, such as those with parents and siblings, conferred by virtue of being born into a family. There are other relationships that develop through interaction, such as those formed with close friends. What makes a relationship important, how individuals within this ring are to behave and how they are to be treated by the person creates specific beliefs, values and principles housed in this ring.

The Societal Ring contains two domains. One, relationships not deemed to be significant by the person will be placed in their Relational Ring. Peripheral relationships that fall into this category include acquaintances, co-workers and may even include family members the individual may not be as intimate with. Also associated with this domain are the specific societal beliefs, values and principles that determine how individuals within this ring are to behave and how they are to be treated by the person. Two, the Societal Ring contains enculturated roles, responsibilities and expectations that the person is bound to by virtue of their being a part of a specific society. It obliges the person to comply with legal, ethical, sociocultural and societal standards and also confers them their basic rights within the society. The size of this ring is dependent on the number of relationships contained within the ring, and its depth is dependent upon the amount of influence the societal element has over the other rings. For physicians, for example, the Societal Ring establishes acceptable standards of practice, desirable work ethic and satisfactory codes of conduct, and captures society's support and considerations for ICU physicians.

Perhaps more significantly, each ring contains specific values, beliefs, principles and expectations that come together within the Individual Ring and influence preferences, motivations, decisions and biases, thoughts, and actions. This highlights the interrelatedness of the rings and the central role of the Individual Ring. Concurrently, changing conditions (Barr and Cacciatore 2008), evolving contextual (Wei et al. 2016), existential, personal, relational and societal considerations also impact the individual's thoughts and actions. This underlines the importance of the RToP's ability to capture changes in thinking, coping (Dyregrov and Gjestad 2011; Youngblut et al. 2017), needs and motivations in ICU physicians, provides an explanation for their decisions (Votta et al. 2001) and actions (Rosenblatt 2000) which will then guide their timely, personalised and targeted support (Braun and Berg 1994). 


\section{Impact of Death and Dying on Doctors}

Several studies (Brooks et al. 2017a, 2017b, Çobanoğlu and Algıer 2004, Hamric and Blackhall 2007, Henrich et al. 2016, Trankle 2014) report that caring for dying patients cause moral, emotional and psychological distress and even disagreements amongst ICU physicians. These studies lend weight to the notion that caring for the dying in the ICU has wide ranging effects upon physicians. Concurrently, evidence of adaptations made to thinking, preferences, decision-making and conduct suggests that facing death and dying influences the beliefs, values and principles contained within each ring, ultimately impacting how the physician perceives their selfhood. For example, in a systematic scoping review of physicians caring for terminally ill children (Ngiam et al. 2021), physicians may struggle to rationalise harsh realities of their patient's situation with their religious beliefs which could potentially lead to a stronger or weaker belief in the afterlife, causing their Innate Ring to shift as their spiritual views change. Due to the interrelatedness of the rings, such a change could cause a ripple effect in the Individual, Relational and Societal Rings, and potentiallya drastic change to their personhood.

\section{Catalysts}

Experiences that instigate changes to a physician's personhood due to ethical, moral or legal challenges to prevailing beliefs, values and principles contained within their Innate, Individual, Relational and/or Societal Rings are referred to as "catalysts". For example, an ICU physician who holds strongly to the sanctity of life by virtue of their religious principles (Innate Ring) may face immense dissonance when confronted with their professional duty to withhold or withdraw life-sustaining treatment deemed clinically futile (Societal Ring).

In order to visualise how events surrounding a patient's dying may serve as a catalyst inciting conflicts within the ICU physician, we consider the scenario of Physician A:

Physician A is a respiratory physician who first diagnosed Patient $X$, a 75 year old man, with chronic pulmonary disease from smoking. About 6 months ago, Physician A diagnosed Patient X with Stage 4 metastatic lung cancer and referred him to an oncologist. Given his long association with Patient X, Physician A continued to review him. About a month ago, Patient $X$ was told his cancer was progressing despite chemotherapy.

Patient X had sought Physician A's advice on how to proceed, knowing that his treatment options were limited. Patient $X$ then explained that he had found the chemotherapy very debilitating and was keen to stop all treatment and wanted to focus on maximising the quality of his remaining life. He had come to seek Physician A's advice on this matter. He agreed for a Home Hospice Care referral and told Physician A that he wanted to spend his remaining days away from the hospital and in the company of his three children who lived 
abroad. He was looking forward to seeing his first grandson born to his eldest son. Physician A helped Patient X complete an Advanced Care Plan (ACP) and informed the oncologist of the patient's desire to stop treatment.

Unfortunately, the next day, the borders of the country where Patient X's children were living were closed due to efforts to curb the spread of COVID-19, leaving his two sons unable to secure outbound flights home. Fortunately, Patient $X$ 's youngest daughter who was on a sailing holiday in Europe was already on-route and arrived just before the borders of Patient X's country were sealed. She did however have to serve a two-week self-isolation period in a local government facility. Unfortunately, she tested positive for COVID-19 and remained at the facility for 38 days.

$A$ week after she completed her self-isolation, Patient $X$ became ill and was taken to hospital with a severe case of pneumonia. After Patient X desaturated in the general ward, he was transferred to the ICU. Physician A broached the topic of Patient X's ACP with his daughter and explained that, although he was in the ICU, he would not be mechanically ventilated should he deteriorate further. In that moment, Patient X's daughter pleaded with Physician A to "do everything and anything possible" to save her father. The daughter refused to acknowledge Patient X's ACP since it had been made before the COVID-19 restrictions were put in place and done in the belief that he could at least see his grandson. Patient X's daughter threatened legal action to have the ACP set aside arguing that it had been made without due consideration of the impact of the COVID-19 restrictions. Furthermore, she argued that now that the travel limitations had eased, a time-trial of ventilation should be allowed to buy time for her brothers to visit.

Here, Patient X's situation is considered a catalyst that brings forth numerous conflicts for Physician A. Firstly, Physician A recognises that whilst Patient X had made the ACP before the COVID-19 travel restrictions, he acknowledges that it was nonetheless a prior arrangement made (Societal Ring). In addition, he feels conflicted as he understands that Patient $\mathrm{X}$ would want to be able to see his children and grandson before he dies (Individual Ring). Furthermore, the ability of Physician A to address these conflicts are compromised by his own staunch religious beliefs in the sanctity of life (Innate Ring). The sheer incongruity between the beliefs, values and principles he upholds in the various Rings of Personhood leaves Physician A feeling deeply unsettled (Fig. 2).

\section{Internal Constituents and External Factors}

Understanding the internal constituents and external factors at play is crucial to understanding how Physician A may address this catalyst and its resultant conflicts.

To clarify, internal constituents include Physician A's beliefs, values, principles and moral considerations in addition to the roles, responsibilities and duties that may arise from his relational, social and professional affiliations. These are already encapsulated in the four rings of the RToP. 


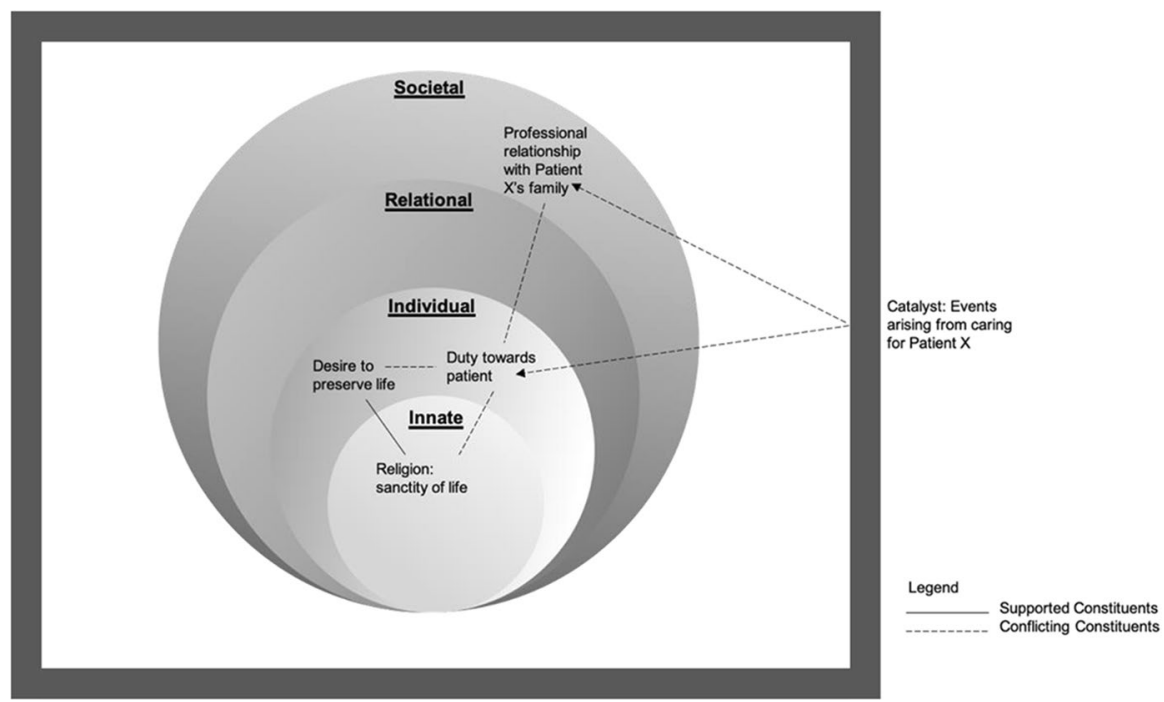

Fig. 2 Catalyst interacting with Physician A's Rings. Conflicting and supporting constituents that interact and affect Physician A's Rings

External factors, on the other hand, are contextual factors which throw the status quo within and between the rings into disarray. They remain outside of Physician A's locus of control. Such external factors can be very real, including a poor working environment, poor intercollegial support and the lack of timely, personalised, accessible support at the institutional level, and the lack of rotations or roles outside of their ICU clinical duties to recuperate, reflect, process their ICU experiences and receive support if required(Kuek et al. 2020).

In this instance, out of the ordinary circumstances have been brought on by the COVID-19 crisis. More specifically, external factors immediately impacting the physician's internal constituents would be the resource limitations posed by the outbreak and the pursuant triaging of ICU beds. Within the context of triaging in the pandemic, Patient X would not be a candidate for ventilatory support in ICU. Whilst such considerations are outside the locus of influence for the physician, they will nonetheless impact the physician keenly as they bear down on his internal constituents and problematise prior standards and norms by offering unprecedented scenarios with its own urgent set of needs and requirements. With different internal constituents affected and different external factors at large, it is clear that catalysts may have different effects on different people at different time points.

\section{Addressing Internal Constituents}

A physician may address conflicts between internal constituents through prioritisation and/or reframing. 


\section{Prioritisation}

When faced with a catalyst that leads to conflict between constituent parts (beliefs, values and/or principles) within and between rings, physicians may choose to prioritise and place greater importance on one constituent over another. For example, Physician A may resist initiation of ventilatory support for Patient $\mathrm{X}$, despite his daughter's wishes, based on the belief and professional stance that patients should have autonomy over their own treatment.

\section{Reframing}

Physician A could reframe competing constituents by altering his perspective of the situation at hand and viewing the decision to withhold ICU interventions as respecting the patient's wishes. This would allow Physician A to meet his obligation to reduce suffering and, in the likelihood that the patient will not regain consciousness to see his children, abide by the ethical principle of beneficence as ICU treatment would be futile.

\section{Dyssynchrony}

In prioritising one set of beliefs, values and principles over another and reframing perspectives, the physician may however find that the "solution" achieved is still in conflict with other constituents, particularly those contained in other rings. Whilst the different rings may offer mutual support for one another, this may not be the case if conflicts persist and are only partially resolved or poorly resolved. Conflicts that continue to persist between rings are referred to as "dyssynchrony" and may result in sharp and prolonged moral, spiritual, emotional and psychological distress. For the ICU physician, such dyssynchrony may bear severe repercussions on their decision-making and functioning as efficacious healthcare professionals with vulnerable and dependent patients under their care.

In prioritising the specific beliefs, values and principles that arise from his professional role and responsibilities, Physician A experiences a greater chasm within his Innate Ring which upholds the sanctity of life and his Societal Ring which sees him desiring to dutifully support his patient's family as best he can. As such, left unchecked, poor resolution of these issues will lead to dyssynchrony between the rings (Fig. 3). It is also evident that in addressing these conflicts, the physician must be mindful of the longitudinal and holistic effects of finding such solutions. It is further suggested that addressing or resolving dyssynchrony is key to supporting these ICU physicians.

Indeed, in discussing the prioritisation and reframing of internal constituents, the impact of external factors become apparent. A tired, emotionally drained physician at the end of a busy week at work confronted with a difficult issue involving 


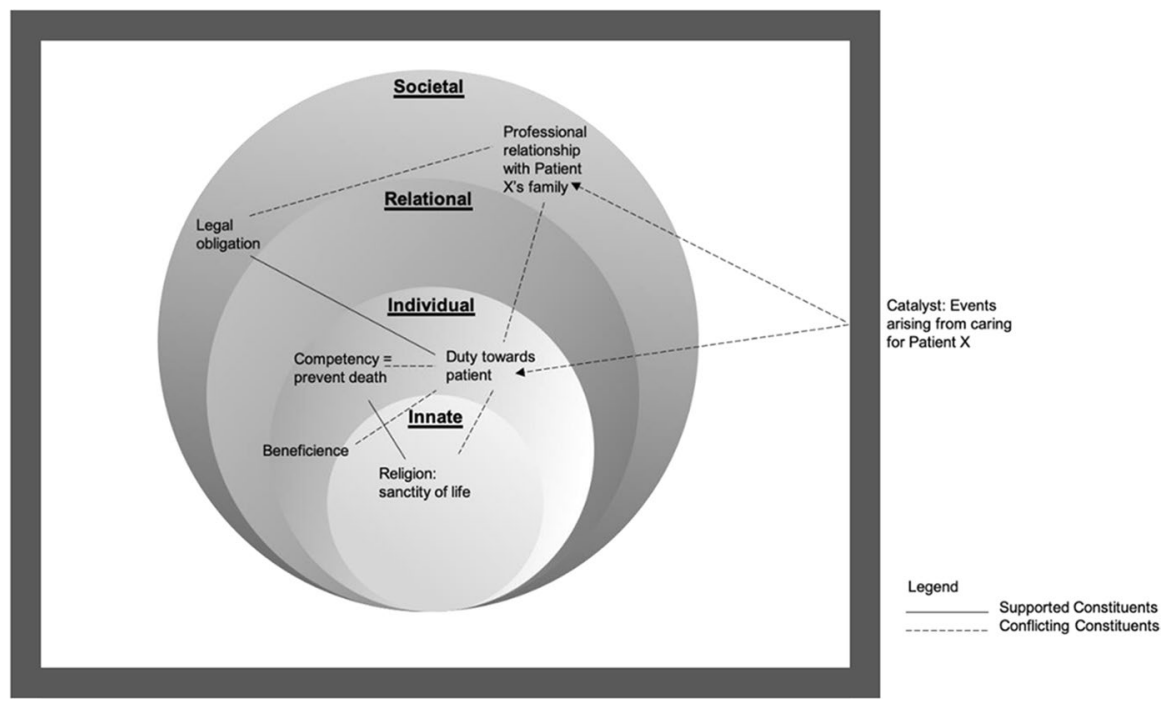

Fig. 3 "Dyssynchrony" in Physician A's Rings of Personhood. Dyssynchrony due to supporting and conflicting constituents within Physician A's Rings

persons that they can "relate to" will likely influence their ability to adequately address these conflicts and temper or resolve the dyssynchrony which may follow.

Fortunately, a good support system surrounds Physician A. In discussing the situation with a colleague and friend, Physician A realises that disregarding patient autonomy has great ethical and legal implications upon his professional and personal beliefs, values and principles. He reasons, and is supported by his colleague and friend, to see that whilst he shared a bond with the patient, this bond may have clouded his decision-making process. Through an open conversation with his religious leaders and deeply reflecting on Patient X's wishes, Physician A acknowledges that his personal conviction to sustain and prolong life in all circumstances may be detrimental to his patient's quality of life. As such, he knows that he has to honour the ACP. Having arrived at a firm decision and resolving the conflict within (Fig. 4), Physician A moves towards a state of synchrony.

Here, a positive influence of external factors is evident - that there are clear benefits to having timely, personalised and holistic clinical, professional and individualised collegial support. A nurturing working environment, opportunities to seek support, and time to reflect was key to supporting Physician A in finding synchrony between his Rings of Personhood.

\section{Buffers}

It is also likely that similar situations in the future will have less of a detrimental impact upon Physician A. Indeed, an ICU physician who is fortified with prior experience and well supported by colleagues and members of their Relational Ring 


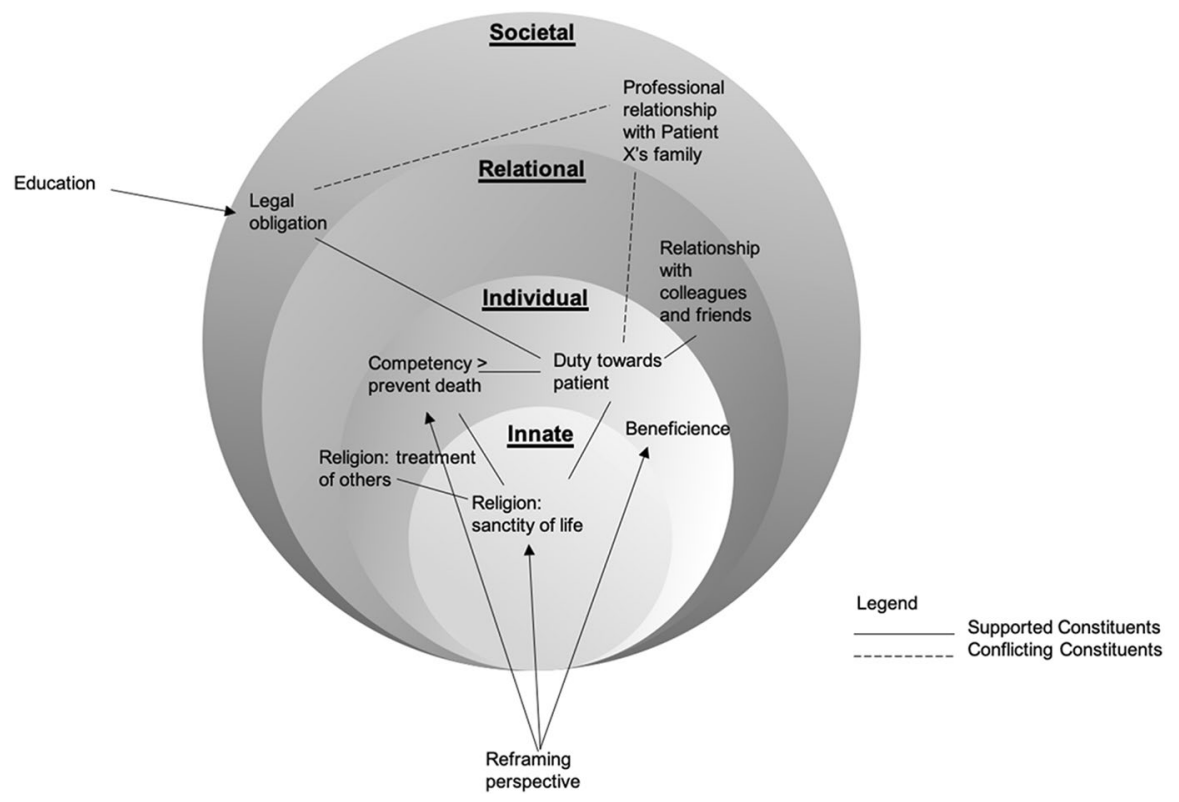

Fig. 4 Physician A's movement from dyssynchrony to synchrony. Movement from dyssynchrony to synchronny within Physician A's Rings

will be better able to address catalysts even before they create conflict or dyssynchrony. Termed as "buffers", we suggest that experience, access to support, time to process, reflect and inculcate lessons learnt in a supportive working environment are in part the basis for the physician's ability to address catalysts, resolve conflicts and develop resilience against them. Notably, buffers not only emerge from external factors but also build upon internal constituents of the rings and may be present in all rings.

\section{Reconceiving the RToP}

The presence of catalysts, conflicts, prioritisation and reframing, external factors and internal constituents, buffering, synchrony and dyssynchrony highlight that concepts of personhood evolve and require review.

To begin, the data suggests that concepts of personhood are not solely concerned with sustaining concepts of "what makes you, you" within the settings of dementia and palliation sedation (Buron 2008; Radha Krishna et al. 2015; Radha Krishna and Alsuwaigh 2015). Rather, it involves the maintaining of a coherent and authentic representation of one's selfhood in "peace time" and in precarious climates where incoming catalysts may incite conflict and dyssynchrony between the various aspects of an individual's concept of personhood, in turn, is determined by the presence and quality of buffers. The use of 
spheres here serve to underline the need to consider the various critical aspects of personhood as identified in this paper (Fig. 5).

These layered spheres reiterate the intertwined nature of the previous rings by maintaining a porous border between it and other layers. These porous borders explain the occurrence of the "ripple effect" when conflict arises between the rings/ spheres and predispose dyssynchrony. Concurrently, these porous boundaries facilitate support for the rings/spheres in conflict as seen when Physician A's colleagues and religious leaders who occupy a place in his Societal Ring/Sphere help address the dyssynchrony between his religious beliefs (Innate Ring/Sphere) and professional responsibilities (Societal and Individual Ring/Spheres). The porous borders may see the outer layers of the Relational Ring/Sphere, in some circumstances, shift into the Societal Ring/Sphere and vice versa.

Changes in the ring/spheres due to conflict will see elements of internal constituents move further away from the sphere's centre as a result of being accorded

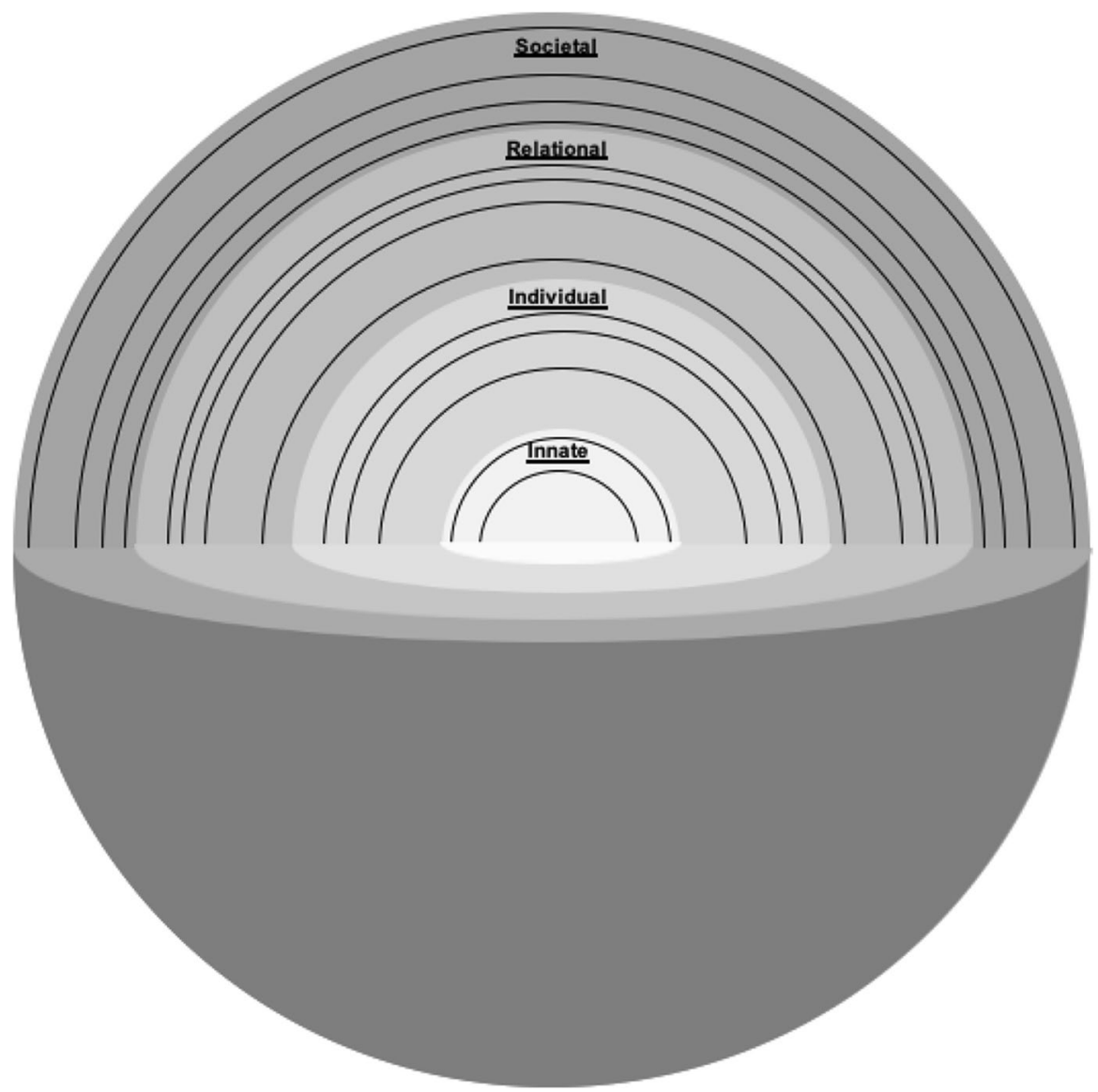

Fig. 5 Spheres of personhood. Moving from rings to the spheres of personhood 
a lower level of importance or ranking. These elements may also reduce in size if associated buffers are attenuated.

\section{Conclusion}

Conceiving the effects of caring for dying patients in ICU from evidence-based data (Kuek et al. 2020) through the lens of the RToP emphasises that the impact of working in the ICU has wide ranging effects upon physicians. Psychological, emotional, academic, research, administrative, personal and professional matters may come together to impact the Innate, Individual, Relational and Societal Rings of their RToP, eventually shifting their needs, values, thinking, motivations and actions with ripple effects.

Thus, from a practical perspective, acknowledging the effects of caring for patients in ICU from a holistic perspective merely foregrounds the need for a concerted effort to comprehensively support clinicians. This framework provides a means of guiding support for ICU physicians at a timely, personalised and targeted manner on two levels - on a personal level, to educate individuals in addressing any conflicts between their internal constituents through prioritisation and reframing, and on an institutional level, to aid host organisations in establishing supportive measures, such as role rotations, timely conflict management interventions, avenues for physicians to share their emotions and experiences. These will help to reduce instances of dyssynchrony, address negative external factors, enhance buffers and ensure a nurturing work environment for ICU physicians.

Understanding the impact of dyssynchrony as a source of internal strife stemming from poor support for ICU physicians also underscores the need to determine how to better assess their needs. Here, the spheres of the RToP may serve as the basis for a longitudinal assessment tool. Such assessments need to be supported by use of portfolios and individualised mentoring processes that provide personalised, appropriate, specific, timely, accessible and long-term support. This would be especially useful for junior physicians in ICU.

The provision of such care for the ICU physician is particularly pertinent whilst the COVID-19 situation rages on and healthcare professionals continue to fight seemingly inextinguishable fires. It is our hope that this paper will help institutions and physicians alike recognise how this striving can take its toll on the firefighter and set out to better equip and protect them from the fire itself.

Acknowledgements The authors would like to dedicate this paper to the late Dr S. Radha Krishna whose advice and ideas were integral to the success of this study. The authors would like to thank the anonymous reviewers whose advice and feedback greatly improved this manuscript.

Author Contribution NPXC, JLC, CYH, LXLN, JTYK, NHAK, ABHMA, YTO, MC, ASIL, AMCC, $\mathrm{SM}$, and LKRK were involved in data curation, formal analysis, investigation, preparing the original draft of the manuscript, and reviewing and editing the manuscript.

All authors have read and approved the manuscript.

Availability of Data and Materials All data generated or analysed during this study are included in this published article and its supplementary information files. 


\section{Declarations}

Ethics Approval NA.

Competing Interests All authors declare no competing interests.

Open Access This article is licensed under a Creative Commons Attribution 4.0 International License, which permits use, sharing, adaptation, distribution and reproduction in any medium or format, as long as you give appropriate credit to the original author(s) and the source, provide a link to the Creative Commons licence, and indicate if changes were made. The images or other third party material in this article are included in the article's Creative Commons licence, unless indicated otherwise in a credit line to the material. If material is not included in the article's Creative Commons licence and your intended use is not permitted by statutory regulation or exceeds the permitted use, you will need to obtain permission directly from the copyright holder. To view a copy of this licence, visit http://creativecommons.org/licen ses/by/4.0/.

\section{References}

Almansour, I., J. Seymour, and A. Aubeeluck. 2019. Staff perception of obstacles and facilitators when providing end of life care in critical care units of two teaching hospitals: A survey design. Intensive and Critical Care Nursing 53: 8-14. https://doi.org/10.1016/j.iccn.2019.04.003.

Amati, Rebecca, and Annegret F. Hannawa. 2014. Relational Dialectics Theory: Disentangling physicianperceived tensions of end-of-life communication. Health Communication 29 (10): 962-973. https:// doi.org/10.1080/10410236.2013.815533.

Asch, D.A., J. Hansen-Flaschen, and P.N. Lanken. 1995. Decisions to limit or continue life-sustaining treatment by critical care physicians in the United States: Conflicts between physicians' practices and patients' wishes. American Journal of Respiratory and Critical Care Medicine 151 (2 Pt 1): 288-292. https://doi.org/10.1164/ajrccm.151.2.7842181.

Aslakson, Rebecca A., Rhonda Wyskiel, Dauryne Shaeffer, Marylou Zyra, Nita Ahuja, Judith E. Nelson, and Peter J. Pronovost. 2010. Surgical intensive care unit clinician estimates of the adequacy of communication regarding patient prognosis. Critical Care 14 (6): R218. https://doi.org/10.1186/ cc9346.

Baggs, Judith Gedney, Madeline H. Schmitt, Thomas J. Prendergast, Sally A. Norton, Craig R. Sellers, Jill R. Quinn, and Nancy Press. 2012. Who is attending? End-of-life decision making in the intensive care unit. Journal of Palliative Medicine 15 (1): 56-62. https://doi.org/10.1089/jpm.2011.0307.

Barnett, Michael D., Beverly R. Williams, and Rodney O. Tucker. 2016. Sudden advanced illness: An emerging concept among palliative care and surgical critical care physicians. American Journal of Hospice \& Palliative Care 33 (4): 321-326. https://doi.org/10.1177/1049909114565108.

Barr, Peter, and Joanne Cacciatore. 2008. Personal fear of death and grief in bereaved mothers. Death Studies 32 (5): 445-460. https://doi.org/10.1080/07481180801974752.

Begley, Sharon. 2020. A plea from doctors in Italy: to avoid Covid-19 disaster, treat more patients at home. Stat News, 21 March 2020. https://www.statnews.com/2020/03/21/coronavirus-plea-fromitaly-treat-patients-at-home/. Accessed 11 Oct 2021.

Braun, Mildred J., and Dale H. Berg. 1994. Meaning reconstruction in the experience of parental bereavement. Death Studies 18 (2): 105-129. https://doi.org/10.1080/07481189408252647.

Brooks, Laura Anne, Elizabeth Manias, and Patricia Nicholson. 2017a. Barriers, enablers and challenges to initiating end-of-life care in an Australian intensive care unit context. Australian Critical Care 30 (3): 161-166. https://doi.org/10.1016/j.aucc.2016.08.001

Brooks, Laura Anne, Elizabeth Manias, and Patricia Nicholson. 2017b. Communication and decisionmaking about end-of-life care in the intensive care unit. American Journal of Critical Care 26 (4): 336-341. https://doi.org/10.4037/ajcc2017774.

Buron, Bill. 2008. Levels of personhood: A model for dementia care. Geriatric Nursing 29 (5): 324-332. https://doi.org/10.1016/j.gerinurse.2007.11.001. 
Chikhladze, Nane, Elene Janberidze, Mariam Velijanashvili, Nikoloz Chkhartishvili, Memed Jintcharadze, Julia Verne, and Dimitri Kordzaia. 2016. Mismatch between physicians and family members views on communications about patients with chronic incurable diseases receiving care in critical and intensive care settings in Georgia: A quantitative observational survey. BMC Palliative Care 15: 63. https://doi.org/10.1186/s12904-016-0135-2.

Çobanoğlu, Nesrin, and Lale Algıer. 2004. A qualitative analysis of ethical problems experienced by physicians and nurses in intensive care units in Turkey. Nursing Ethics 11 (5): 444-458. https://doi.org/ 10.1191/0969733004ne723oa.

Cohen, Simon, Charles Sprung, Peter Sjokvist, Anne Lippert, Bara Ricou, Mario Baras, Seppo Hovilehto, Paulo Maia, Dermot Phelan, Konrad Reinhart, Karl Werdan, Hans-Henrik Bulow, and Tom Woodcock. 2005. Communication of end-of-life decisions in European intensive care units. Intensive Care Medicine 31 (9): 1215-1221. https://doi.org/10.1007/s00134-005-2742-x.

Daly, Barbara J., Sara L. Douglas, Elizabeth O’Toole, James Rowbottom, Alan Hoffer, Amy R. Lipson, and Christopher Burant. 2018. Complexity analysis of decision-making in the critically ill. Journal of Intensive Care Medicine 33 (10): 557-566. https://doi.org/10.1177/0885066616 678394.

Donnelly, Sinead M., and A. Psirides. 2015. Relatives' and staff's experience of patients dying in ICU. QJM 108 (12): 935-942. https://doi.org/10.1093/qjmed/hcv059.

Dyregrov, Atle, and Rolf Gjestad. 2011. Sexuality following the loss of a child. Death Studies 35 (4): 289-315. https://doi.org/10.1080/07481187.2010.527753.

Engelhart, Katie, and Dominic Smith. 2020. Who gets a ventilator? New York Times, 14 April 2020. https://www.nytimes.com/2020/04/14/opinion/coronavirus-ventilator-rationing.html. Accessed 11 Oct 2021.

Festic, Emir, Michael E. Wilson, Ognjen Gajic, Gavin D. Divertie, and Jeffrey T. Rabatin. 2012. Perspectives of physicians and nurses regarding end-of-life care in the intensive care unit. Journal of Intensive Care Medicine 27 (1): 45-54. https://doi.org/10.1177/0885066610393465.

Gutierrez, Karen M. 2012. Advance directives in an intensive care unit: Experiences and recommendations of critical care nurses and physicians. Critical Care Nursing Quarterly 35 (4): 396-409. https://doi.org/10.1097/CNQ.0b013e318268fe35.

Hamric, Anne B., and Leslie J. Blackhall. 2007. Nurse-physician perspectives on the care of dying patients in intensive care units: Collaboration, moral distress, and ethical climate. Critical Care Medicine 35 (2): 422-429. https://doi.org/10.1097/01.Ccm.0000254722.50608.2d.

Hawryluck, Laura A., William R. Harvey, Louise Lemieux-Charles, and Peter A. Singer. 2002. Consensus guidelines on analgesia and sedation in dying intensive care unit patients. BMC Medical Ethics 3: 3. https://doi.org/10.1186/1472-6939-3-3.

Henrich, Natalie J., Peter M. Dodek, Lynn Alden, Sean P. Keenan, Steven Reynolds, and Patricia Rodney. 2016. Causes of moral distress in the intensive care unit: A qualitative study. Journal of Critical Care 35: 57-62. https://doi.org/10.1016/j.jcrc.2016.04.033.

Hough, Catherine L., Leonard D. Hudson, Antonio Salud, Timothy Lahey, and J. Randall Curtis. 2005. Death rounds: End-of-life discussions among medical residents in the intensive care unit. Journal of Critical Care 20 (1): 20-25. https://doi.org/10.1016/j.jcrc.2004.09.006.

Jensen, Hanne Irene, Jette Ammentorp, Helle Johannessen, and Helle Ørding. 2013. Challenges in end-of-life decisions in the intensive care unit: An ethical perspective. Journal of Bioethical Inquiry 10 (1): 93-101. https://doi.org/10.1007/s11673-012-9416-5.

Kuek, Joshua Tze Yin, Lisa Xin Ling Ngiam, Nur Haidah Ahmad Kamal, Jeng Long Chia, Natalie Pei Xin Chan, Ahmad Bin Hanifah Marican Abdurrahman, Chong Yao Ho, Lorraine Hui En Tan, Jun Leng Goh, Michelle Shi Qing Khoo, Yun Ting Ong, Min Chiam, Annelissa Mien Chew Chin, Stephen Mason, and Lalit Kumar Radha Krishna. 2020. The impact of caring for dying patients in intensive care units on a physician's personhood: A systematic scoping review. Philosophy, Ethics, and Humanities in Medicine 15: 12. https://doi.org/10.1186/s13010-020-00096-1.

Laurent, Alexandra, Magalie Bonnet, Gilles Capellier, Pierre Aslanian, and Paul Hebert. 2017. Emotional impact of end-of-life decisions on professional relationships in the ICU: An obstacle to collegiality? Critical Care Medicine 45 (12): 2023-2030. https://doi.org/10.1097/ccm.00000 00000002710 .

McAndrew, Nathalie S., and Jane S. Leske. 2015. A balancing act: Experiences of nurses and physicians when making end-of-life decisions in intensive care units. Clinical Nursing Research 24 (4): 357-374. https://doi.org/10.1177/1054773814533791. 
Mehter, Hashim M., Jessica B. McCannon, Jack A. Clark, and Renda Soylemez Wiener. 2018. Physician approaches to conflict with families surrounding end-of-life decision-making in the intensive care unit. A qualitative study. Annals of the American Thoracic Society 15 (2): 241-249. https://doi.org/10.1513/AnnalsATS.201702-105OC.

Monteiro, Mayla Cosmo, Andrea Seixas Magalhães, Terezinha Féres-Carneiro, and Rebeca Nonato Machado. 2016. Terminality in the ICU: The emotional and ethical dimensions of the medical care of the intensivist. Psicologia Em Estudo 21 (1): 65-75. https://doi.org/10.4025/psicolestud. v21i1.28480.

Ngiam, Lisa Xin Ling, Yun Ting Ong, Jun Xuan Ng, Joshua Tze Yin Kuek, Jeng Long Chia, Natalie Pei Xin Chan, Chong Yao Ho, Ahmad Bin Hanifah Marican Abdurrahman, Nur Haidah Ahmad Kamal, Clarissa Wei Shuen Cheong, Cheng Han Ng, Xiu Hui Tan, Lorraine Hui En Tan, Annelissa Mien Chew Chin, Stephen Mason, Muhammad Raihan Jumat, Min Chiam, Lalit Kumar Radha Krishna. 2021. Impact of caring for terminally ill children on physicians: A systematic scoping review. American Journal of Hospice and Palliative Medicine 38 (4): 396-418. https://doi.org/10.1177/1049909120950301.

Nordgren, Lena, and Henny Olsson. 2004. Palliative care in a coronary care unit: A qualitative study of physicians' and nurses' perceptions. Journal of Clinical Nursing 13 (2): 185-193. https://doi. org/10.1111/j.1365-2702.2004.00816.x.

Oliver, David. 2020. The intensive care unit is not the best place for all Covid-19 patients. Financial Times, 19 April 2020. https://www.ft.com/content/2f170f9e-7d98-11ea-b0fb-13524ae1056b. Accessed 11 Oct 2021.

Ouyang, Helen. 2020a. At the front lines of coronavirus, turning to social media. New York Times, 18 March 2020. https://www.nytimes.com/2020/03/18/well/live/coronavirus-doctors-facebook-twitt er-social-media-covid.html. Accessed 11 Oct 2021.

Ouyang, Helen. 2020b. I'm an E.R. Doctor in New York. None of us will ever be the same. New York Times, 14 April 2020. https://www.nytimes.com/2020/04/14/magazine/coronavirus-er-doctor-diarynew-york-city.html. Accessed 11 Oct 2021.

Pattison, Nathalie, Susan M. Carr, Chris Turnock, and Shelley Dolan. 2013. 'Viewing in slow motion': patients', families', nurses' and doctors' perspectives on end-of-life care in critical care. Journal of Clinical Nursing 22 (9-10): 1442-1454. https://doi.org/10.1111/jocn.12095.

Phua, Jason, Li Weng, Lowell Ling, Moritoki Egi, Chae-Man Lim, Jigeeshu Vasishtha Divatia, Babu Raja Shrestha, Yaseen M. Arabi, Jensen Ng, Charles D. Gomersall, Masaji Nishimura, Younsuck Koh, and Bin Du, for the Asian Critical Care Clinical Trials Group. 2020. Intensive care management of coronavirus disease 2019 (COVID-19): Challenges and recommendations. Lancet Respiratory Medicine 8 (5): 506-517. https://doi.org/10.1016/s2213-2600(20)30161-2.

Radha Krishna, Lalit Kumar, and Rayan Alsuwaigh. 2015. Understanding the Fluid Nature of Personhood - the Ring Theory of Personhood. Bioethics 29 (3): 171-181. https://doi.org/10.1111/bioe.12085.

Radha Krishna, Lalit Kumar, and Suat Yee Kwek. 2015. The Changing Face of Personhood at the End of Life: The Ring Theory of Personhood. Palliative and Supportive Care 13 (4): 1123-1129. https:// doi.org/10.1017/S1478951514000686.

Robertsen, Annette, Eirik Helseth, Jon Henrik Laake, and Reidun Førde. 2019. Neurocritical care physicians' doubt about whether to withdraw life-sustaining treatment the first days after devastating brain injury: An interview study. Scandinavian Journal Trauma, Resuscitation and Emergency Medicine 27: 81. https://doi.org/10.1186/s13049-019-0648-9.

Rosenblatt, Paul C. 2000. Protective parenting after the death of a child. Journal of Personal and Interpersonal Loss 5 (4): 343-360. https://doi.org/10.1080/10811440008407851.

Schmidt, Harald. 2020. The way we ration ventilators is biased. New York Times, 15 April 2020. https:// www.nytimes.com/2020/04/15/opinion/covid-ventilator-rationing-blacks.html. Accessed 11 Oct 2020.

Simmonds, Anne. 1996. Decision-making by default: experiences of physicians and nurses with dying patients in intensive care. Human Health Care International 12 (4): 168-172.

Svantesson, Mia, Peter Sjökvist, and Håkan Thorsén. 2003. End-of-life decisions in Swedish ICUs: How do physicians from the admitting department reason? Intensive and Critical Care Nursing 19 (4): 241-251. https://doi.org/10.1016/S0964-3397(03)00055-7.

Trankle, Steven A. 2014. Is a good death possible in Australian critical and acute settings?: physician experiences with end-of-life care. BMC Palliative Care 13: 41. https://doi.org/10.1186/ 1472-684X-13-41. 
Votta, Elizabeth, Renée-Louise Franche, Dalice Sim, Beth Mitchell, Tim Frewen, and Cathy Maan. 2001. Impact of parental involvement in life-support decisions: A qualitative analysis of parents' adjustment following their critically ill child's death. Children's Health Care 30 (1): 17-25. https://doi. org/10.1207/S15326888CHC3001_2.

Wåhlin, Ingrid, Anna-Christina Ek, and Ewa Idvall. 2010. Staff empowerment in intensive care: Nurses' and physicians' lived experiences. Intensive and Critical Care Nursing 26 (5): 262-269. https://doi. org/10.1016/j.iccn.2010.06.005.

Wei, Yan, Quanbao Jiang, and Stuart. Gietel-Basten. 2016. The well-being of bereaved parents in an onlychild society. Death Studies 40 (1): 22-31. https://doi.org/10.1080/07481187.2015.1056563.

Weng, Li, Gavin M. Joynt, Anna Lee, Bin Du, Patricia Leung, Jinming Peng, Charles D. Gomersall, Xiaoyun Hu, and Hui Y. Yap, for the Chinese Critical Care Ethics Group. 2011. Attitudes towards ethical problems in critical care medicine: The Chinese perspective. Intensive Care Medicine 37 (4): 655-664. https://doi.org/10.1007/s00134-010-2124-x.

Workman, Stephen, Patricia McKeever, William Harvey, and Peter A. Singer. 2003. Intensive care nurses' and physicians' experiences with demands for treatment: some implications for clinical practice. Journal of Critical Care 18 (1): 17-21. https://doi.org/10.1053/jcrc.2003.YJCRC4.

Youngblut, JoAnne M., Dorothy Brooten, Joy Glaze, Teresita Promise, and Changwon Yoo. 2017. Parent grief 1-13 months after death in neonatal and pediatric intensive care units. Journal of Loss and Trauma 22 (1): 77-96. https://doi.org/10.1080/15325024.2016.1187049.

Zambrano, Sofia Carolina, Anna Chur-Hansen, and Brian Crawford. 2012. On the emotional connection of medical specialists dealing with death and dying: A qualitative study of oncologists, surgeons, intensive care specialists and palliative medicine specialists. BMJ Supportive \& Palliative Care 2 (3): 270-275. https://doi.org/10.1136/bmjspcare-2012-000208.

Publisher's Note Springer Nature remains neutral with regard to jurisdictional claims in published maps and institutional affiliations.

\section{Authors and Affiliations}

\section{Natalie Pei Xin Chan ${ }^{1,2}$. Jeng Long Chia ${ }^{1,2}$. Chong Yao Ho ${ }^{1,2} \cdot$ Lisa Xin Ling Ngiam ${ }^{1,2}$. Joshua Tze Yin Kuek ${ }^{1,2}$ • Nur Haidah Binte Ahmad Kamal,2 . Ahmad Bin Hanifah Marican Abdurrahman ${ }^{1,2} \cdot$ Yun Ting Ong ${ }^{1,2} \cdot$ Min Chiam $^{3}$. Alexia Sze Inn Lee ${ }^{3}$. Annelissa Mien Chew Chin ${ }^{4}$ Stephen Mason ${ }^{5,6}$. Lalit Kumar Radha Krishna 1,2,3,5,6,7,8,9 \\ * Lalit Kumar Radha Krishna \\ lalit.radha-krishna@liverpool.ac.uk}

1 Yong Loo Lin School of Medicine, National University of Singapore, Singapore

2 Division of Supportive and Palliative Care , National Cancer Centre Singapore, Singapore

3 Division of Cancer Education, National Cancer Centre Singapore, Singapore

4 Medical Library, National University of Singapore Libraries, National University of Singapore, Singapore

5 Palliative Care Institute Liverpool, Academic Palliative \& End of Life Care Centre, University of Liverpool, Liverpool, UK

6 Cancer Research Centre, University of Liverpool, Liverpool, UK

7 Palliative Care Centre for Excellence in Research and Education (PalC), Singapore

8 Centre for Biomedical Ethics, National University of Singapore, Singapore

9 Duke-NUS Graduate Medical School, Singapore 